${ }^{1}$ Department of Restorative Dentistry, School of Dentistry of Ribeirão Preto, University of São Paulo (USP), Ribeirão Preto, SP, Brazil.
Corresponding author: Juliana Jendiroba Faraoni Avenida do Café, $s / n^{\circ}$, Ribeirão Preto, Brazil Phone +55(16)3315-4016 jujfaraoni@forp.usp.br (iD) https://orcid.org/0000-0003-0945-4028

Received: March 22, 2019 Accepted: October 01, 2019

\section{Color stability of nanohybrid composite resins in drinks}

\author{
Juliana Jendiroba Faraoni ${ }^{1, *}$, Isabela Barbosa \\ Quero' ${ }^{1}$ Lívia Semedo Schiavuzzoํㄹ Regina Guenka \\ Palma - Dibb ${ }^{1}$
}

Aim: The objective of this study was to evaluate the effects of solutions on the color stability of nanohybrid composite resins. Methods: The experimental sample consisted of 90 composite specimens (Beautifil II; Z350XT; Premisa), divided into three subgroups $(n=10)$ according to the solutions (matte tea; lemon flavor isotonic drink; artificial saliva). The specimens were immersed in the solutions ( $5 \mathrm{~mL} / \mathrm{specimen}$ ) while stirring for 5 minutes, four times a day, with 1-hour intervals, repeated for 15 days. The color of the specimens was analyzed before (baseline) and after the $15^{\text {th }}$ day of cycling using the CIELAB system. Data were analyzed using the two-way analysis of variance (ANOVA) and Tukey's test $(a=5 \%)$. Results: Different behaviors were observed among resins. Beautifil II presented the highest color change $(\Delta E=4.18)$ and less color stability, statistically different from the others $(p<.05)$. The solutions also presented different behaviors. The lemon flavor isotonic drink $(\Delta E=3.95)$ promoted the highest color change, statistically different from saliva $(\Delta E=2.75 ; p<.05)$. The interaction between Beautifil II and isotonic drink became even more evident and significant $(p<.05)$. Conclusion: The isotonic drink was the solution that most affected the resins, and Beautifil II presented the worst color stability.

Keywords: Composite resins. Nanocomposites. Color. Beverages. 


\section{Introduction}

The demand and advances in the aesthetic area have grown, primarily with an evolution of composite resins in dental practice ${ }^{1,2,3}$. Alteration of color is one of the many reasons for replacement of a composite resin restoration. However, this procedure costs time and money and increases the size of the cavity ${ }^{4}$. Color alteration is attributed to intrinsic discolorations due to physicochemical reactions, such as the quality of the polymer matrix of the resin or the quality of photopolymerization $^{5,6}$. There are also extrinsic discolorations, which are related to biofilm accumulation and staining by adsorption or absorption of pigments, mostly present in drinks and food ${ }^{7,8}$. In addition, other properties should be considered, such as the surface texture, staining agent, exposure time to the pigment, and characteristics of the restoration material ${ }^{4}$.

Some studies ${ }^{3,8-12}$ demonstrated that some drinks, such as tea, red wine, coffee, juice, and soft drinks, are responsible for staining the composite resins to various degrees. Besides these, sports drinks can also influence the stability of color of composite resins $^{13}$. The consumption of this kind of beverage is elevated ${ }^{14}$ due to a new beauty standard that promotes a "new" modern lifestyle with a "healthy diet" and regular exercise, and the researches are not involving this category ${ }^{14}$.

Many modifications have been taking place regarding composite resin to produce a material that is more aesthetic with better polish and good mechanical properties. This change has been occurring in the size of particles, which produce a functional material with nanosized phases called nanocomposites ${ }^{15}$. This change in charged particles and the monomers of the matrix results in a lower polymerization shrinkage, better retention, and better aesthetics ${ }^{1,16}$ without compromising mechanical strength ${ }^{17}$.

Despite this, there are few studies regarding the color stability of these materials. Therefore, the aim of this study was to evaluate the color stability of nanohybrid composite resins in the immersion of different commercial drinks.

\section{Materials and Methods}

\section{Experimental Design}

The experimental sample consisted of 90 resin specimens of composite resin. The specimens were divided into nine groups/subgroups $(n=10)$, considering three composite resins: Beautifil II (Shofu, Kyoto City, Kyoto Prefecture, Japan), Z350XT (3M ESPE, St. Paul, Minnesota, USA), and Premisa (Kerr, Orange, California, USA) and three solutions: matte tea (Leão Alimentos e Bebidas, Fazenda Rio Grande, Paraná, Brazil), lemon flavor isotonic drink (Gatorade - Ambev., Jaguariúna, São Paulo, Brazil; glucose, water, sucrose, sodium chloride, sodium citrate, potassium phosphate, citric acid, and flavoring), and artificial saliva.

This study was conducted using a randomized complete block design. The quantitative response was the color stability $(\Delta E, \Delta L, \Delta a$, and $\Delta b)$. 


\section{Preparation of Specimens}

The composite resins (Table 1) were manipulated according to the manufacturer's instructions and were inserted into cylindrical metal molds of stainless steel (4 mm diameter $\times 2 \mathrm{~mm}$ thickness/height). This insertion was performed in a single increment.

Table 1. Composite resins tested in this study.

\begin{tabular}{|c|c|c|c|c|c|}
\hline $\begin{array}{l}\text { Composite } \\
\text { Resin }\end{array}$ & Matrix & Size of Fillers & $\begin{array}{l}\text { Percentage of } \\
\text { Fillers }\end{array}$ & Filler & Manufacturer \\
\hline Beuatifil II & Bis-GMA, TEGDMA & $10 n m-20 n m$ & $\begin{array}{l}54 \%(\mathrm{~L} / \mathrm{V}) 74 \% \\
(\mathrm{~L} / \mathrm{Wt})\end{array}$ & S-PRG & Shofu Inc \\
\hline Filtek Z350XT & $\begin{array}{c}\text { Bis-GMA, UDMA, } \\
\text { TEGDMA, PEGDMA, } \\
\text { Bis-EMA }\end{array}$ & $\begin{array}{c}4-11 \mathrm{~nm}, 20 \\
\mathrm{~nm}\end{array}$ & $\begin{array}{l}63.3 \% \\
\text { (V)/78.5\% } \\
\text { Weight }\end{array}$ & $\begin{array}{l}\text { Zirconia, silica } \\
\text { cluster (20 } \\
\text { nm) }\end{array}$ & 3M ESPE \\
\hline Primesa & $\begin{array}{c}\text { Bis-GMA, BisEMA, } \\
\text { TEGDMA }\end{array}$ & $0.02 \mu \mathrm{m}$ & $84 \%(\mathrm{Wt})$ & $\begin{array}{c}\text { PPF filler, } \\
\text { Point } 4 \text { filler, } \\
0.02 \mu \mathrm{m}\end{array}$ & Kerr Corp \\
\hline
\end{tabular}

After the insertion, the matrices were covered with a glass slide. Then, an axial load of $500 \mathrm{~g}$ was applied on each specimen for $1 \mathrm{~min}$. This compress created a flat surface and standardized the thickness. After $1 \mathrm{~min}$, the load was removed, and the material was photopolymerized using Kavo Poly Wireless (Kavo do Brasil, Joinville, Santa Catarina, Brazil) through glass with visible light for 20 seconds. The intensity of the visible light was monitored by a radiometer and was maintained at around $1100 \mathrm{~mW} / \mathrm{cm}^{2}$.

After preparation, the specimens were held and stored in artificial saliva in the oven at $37^{\circ} \mathrm{C}\left(+/-1^{\circ} \mathrm{C}\right)$. After $24 \mathrm{~h}$, the specimens were submitted to finishing and polishing phases in a polish machine (Arotec, São Paulo, São Paulo, Brazil) with water sandpaper 600 and 1200 and with 0.3- and 0.05- $\mu$ m alumina suspensions. By the end of these procedures, the specimens were washed with distilled water for $30 \mathrm{~s}$, submerged into distilled water at the ultrasound for 5 min, dried with paper towels, and then immersed in artificial saliva for $24 \mathrm{~h}$ at $37^{\circ} \mathrm{C}$.

\section{Baseline Color Analysis}

Before the cycling, the original color of each specimen was analyzed with a spectrophotometer (Color guide 45/0, PCB 6807 BYK-Gardner GmbH, Geretsried, Bavaria, Germany) on a white background. This handheld portable equipment measures color and gloss attributes simultaneously. The spectro-guide spectrophotometer allows repeatable results using color guide $45 / 0$ and a $4-\mathrm{mm}$ aperture and circumferential illumination. The standard of observation simulated by spectrophotometer follows the CIELAB system, recommended by the Commission Internationale de l'Éclairage (CIE). This consists of two axes: $a^{\star}$ and $b^{\star}$. They have right angles, representing the size of the shade or color. The third axis is the brightness: $L^{\star}$. It is perpendicular to the 
plane with axes $a^{*}$ and $b^{*}$. With this system, any color can be specified by the coordinates $L^{*}, a^{*}$, and $b^{*}$. We activated the spectrophotometer (30 LED lamps) with 10 different colors, arranged in a circular shape, and focused the light beam at $45^{\circ}$ with the material surface. This beam is reflected back at $0^{\circ}$ to the apparatus, and it captures and records the values $L^{\star}, a^{\star}$, and $b^{\star}$ of each sample.

\section{Cycling of Samples}

Specimens of each composite resin were randomly divided into three subgroups. The control group was kept in artificial saliva and the other two experimental groups were submitted into cycling with the selected drink (matte tea or lemon flavor isotonic drink). The drinks were used in their consumption temperature, with matte tea at $40^{\circ} \mathrm{C}$ and the isotonic drink at $4^{\circ} \mathrm{C}$. Temperatures were measured with a digital thermometer.

For 5 min, specimens were immersed in the drinks $(5 \mathrm{ml} /$ specimen) under agitation (Orbital Shaker Table CT-155, Cientec Laboratories Equipment, Piracicaba, São Paulo, Brazil), 4 times a day, with 1-h intervals. Among the cycles, the specimens were immersed in artificial saliva at $37^{\circ} \mathrm{C}\left(+/-1^{\circ} \mathrm{C}\right)$. For the control group, the specimens were kept in an oven at $37^{\circ} \mathrm{C}\left(+/-1^{\circ} \mathrm{C}\right)$ changing the solution daily. These procedures were repeated for $15 \mathrm{~d}$.

\section{Final Color Analysis}

After the cycling period, the color was measured again. The difference between the color results was obtained by calculating $\Delta E^{\star}=\left[\left(\Delta L^{\star}\right) 2+\left(\Delta a^{\star}\right) 2+\left(\Delta b^{\star}\right) 2\right] 12$. The brightness differences of $\Delta L, \Delta a$, and $\Delta b$ were also calculated by the formulas $\Delta L^{*}=L^{*}(t)-I^{\star}(0), \Delta a^{\star}=a^{\star}(t)-a^{\star}(0)$, and $\Delta b^{\star}=b^{\star}(t)-b^{\star}(0)$, where $(t)$ is the time and $(0)$ is the baseline. The color changes were obtained by the values $\Delta E, \Delta L, \Delta a$, and $\Delta b$.

Data were analyzed based on distribution and homogeneity, showing normal (Shapiro-Wilks) and homogeneous (Levene's) results. The analysis of variance (ANOVA) used two criteria (two-way ANOVA: resin and solution) and the Tukey test $(p<.05)$ to distinguish the means.

\section{Results}

\section{Change in Brightness $(\Delta \mathrm{L})$}

For factor composite resins, similar results $(p>$.05) were observed for the three nanohybrid composite resins. However, for the solutions, the lemon flavor isotonic drink was the solution that most affected the specimens, making them clearer, which was a statistically significant difference from the other solutions studied $(p<.05)$. The other two solutions presented similar results $(p>.05)$. In the interaction, the composite Premisa showed no significant difference for the solutions ( $p>$.05; Table 2). The tea solution darkened the Z350 and Beautifil II, and the isotonic drink samples were lighter. 
Table 2. Mean and standard deviation of $\Delta L$ for the different resins and solutions.

\begin{tabular}{lccc}
\hline Composite Resin & Saliva & Tea & Isotonic Drink \\
\hline Premisa & $1.58 \pm 2.40$ a A & $-0.04 \pm 3.22$ a A & $0.44 \pm 1.94$ b A \\
\hline Z350 XT & $1.12 \pm 3.48$ a A & $-1.92 \pm 2.49$ a B & $0.27 \pm 1.27$ b AB \\
\hline Beautifil II & $-0.51 \pm 2.04$ a B & $-0.97 \pm 2.41$ a B & $6.25 \pm 4.78$ a A \\
\hline
\end{tabular}

*Capital letter indicates statistical difference among columns.

Lowercase indicates statistical difference among lines.

\section{Change in Color $(\Delta \mathrm{E})$}

In the color analysis for the composite resin factor, Beautifil II showed the greatest change and presented a statistical difference from the other composites studied $(p<$.05). In turn, Premisa and Z350 were similar $(p>.05)$.

Comparing the solutions, the lemon flavor sports drink caused changes in the composite resin, with a statistically significant difference compared with the other solutions $(p<.05)$. The other two solutions were similar $(p>.05)$. Considering the interaction, only Beautifil II showed significant changes for the isotonic drink $(p<.05$; Table 3$)$.

Table 3. Mean and standard deviation of $\Delta \mathrm{E}$ for the different resins and solutions.

\begin{tabular}{lccc}
\hline Composite Resin & Saliva & Tea & Isotonic Drink \\
\hline Premisa & $2.57 \pm 1.43$ a A & $3.04 \pm 1.84$ a A & $2.53 \pm 1.27$ b A \\
\hline Z350 XT & $3.34 \pm 2.09$ a A & $3.32 \pm 0.93$ a A & $1.98 \pm 0.82$ b A \\
\hline Beautifil II & $2.32 \pm 1.05$ a B & $2.88 \pm 1.65$ a B & $7.35 \pm 3.71$ a A \\
\hline
\end{tabular}

*Capital letter indicates statistical difference among columns.

Lowercase indicates statistical difference among lines.

\section{Changes in $\mathrm{a}^{*}$ and $\mathrm{b}$ *}

Regarding $\triangle a$, the three resins presented different behaviors $(p<.05)$, and the composite resin Z350 demonstrated the most variance. However, statistically, the solutions did not affect the samples ( $p>$.05). In the interaction of the factors, only Beautifil II showed significant changes for the isotonic drink $(p<.05$; Table 4$)$.

Table 4. Mean and standard deviation of $\Delta \mathrm{a}$ for the different resins and solutions.

\begin{tabular}{lccc}
\hline Composite Resin & Saliva & Tea & Isotonic Drink \\
\hline Premisa & $0.28 \pm 0.44$ a A & $0.05 \pm 0.54$ b A & $0.29 \pm 0.38$ a A \\
\hline Z350 XT & $0.50 \pm 0.82$ a A & $1.07 \pm 0.47$ a A & $0.80 \pm 0.63$ a A \\
\hline Beautifil II & $0.02 \pm 0.67$ a A & $-0.05 \pm 0.70$ b AB & $-0.72 \pm 3.71 \mathrm{~b} \mathrm{~B}$ \\
\hline
\end{tabular}

*Capital letter indicates statistical difference among columns.

Lowercase indicates statistical difference among lines. 
In the analysis, the three composites showed similar results for $\Delta b(p>$.05). However, the lemon flavor isotonic drink affected the specimens and demonstrated a distinct behavior from the artificial saliva and matte tea $(p<.05)$. In the interaction, only Beautifil II showed significant changes with the lemon flavor sports drink $(p<.05$; Table 5$)$.

Table 5. Mean and standard deviation of $\Delta b$ for the different resins and solutions.

\begin{tabular}{lccc}
\hline Composite Resin & Saliva & Tea & Isotonic Drink \\
\hline Premisa & $0.43 \pm 0.68$ a A & $-0.33 \pm 1.70$ a A & $-1.38 \pm 1.54$ a A \\
\hline Z350 XT & $-0.22 \pm 1.51$ a A & $-0.34 \pm 1.09$ a A & $-1.22 \pm 0.79$ a A \\
\hline Beautifil II & $-1.00 \pm 1.4$ a A & $-0.43 \pm 2.10$ a A & $-2.12 \pm 1.27$ b B \\
\hline
\end{tabular}

*Capital letter indicates statistical difference among columns.

Lowercase indicates statistical difference among lines.

\section{Discussion}

Consuming sports drinks and teas has increased considerably due to habit changes for a healthier lifestyle ${ }^{13}$. However, these solutions provide erosion and staining in tooth structure and esthetic restorative materials ${ }^{14,18,19}$, and this effect can directly affect patient satisfaction with the color of the restoration ${ }^{20}$. Also, at long term, the association of the consumption of these kind of beverages with toothbrushing can influence on the material's longevity in relation to the contour and coloration ${ }^{3}$.

The perception of color is related to psychological aspects and can be interpreted based on different factors according to the observer's skills. Because of these errors, devices that assist in the evaluation of color were used, and data were obtained using the CIELAB system ${ }^{21,22}$. In several studies ${ }^{23,24}$, the color change is deemed acceptable for values up to $\Delta E=3.3$, determining a threshold for visual perception. In the present study, the three resins showed color alteration after immersion into the tested solutions, including artificial saliva (control). This might have occurred because of the period to which the samples were immersed, since the artificial saliva significantly influences the color stability of restorative materials because of its components and water sorption by the resin matrix ${ }^{3}$. The color alteration can be attributed to intrinsic discolorations due to physicochemical reactions and to the quality of the polymer matrix of the resin or the quality of photopolymerization ${ }^{6}$. It can also be attributed to the extrinsic discolorations, which are related to biofilm accumulation and staining by adsorption or absorption of pigments that are present in food and drinks ${ }^{20}$. Different drinks, such as tea, soda, beer, coffee, and orange juice, can affect the physical and chemical structures of restorative materials ${ }^{17}$. In addition, the oral environment associated with the characteristics of the beverage can influence the discoloration of restorative materials and affect the surface integrity ${ }^{25}$.

Results shows that, among the drinks, isotonic drinks presented the most distinguished results affecting the brightness for axis $a^{*}$ and axis $b^{*}$ of the resins, leading to a significant color change in the composite Beautifil II. Of the solutions, the lemon 
flavor isotonic drink most altered the stability of the tested composites, that can be explained because of the acid $\mathrm{pH}$ of these beverage ${ }^{19}$. Although tea is considered one of the most decolorizing beverages ${ }^{26}$, its consumption is still very high in the population. In our study, it did not cause a major alteration of color in the composite resins tested, presenting similar behavior to saliva. In this study, artificial saliva did not provoke major color alteration either ${ }^{20}$.

In the analysis of $\triangle E$ values, Premisa presented better stability to immersion in various solutions, and any solution promoted color alteration up to 3.3, since $\Delta E$ values up to 3.3 is considered clinically accepted ${ }^{3}$. Filtek Z350 XT and Beautifil II showed a higher color change when compared to Premisa. Beautifil II showed higher values $(\Delta E=7.35)$ when immersed into the sports drink (isotonic drink), indicating clinically visible change in color. One hypothesis for these results is that the acid solution may have degraded resin surface, interfering with the light reflection. This fact can be observed by the brightness in the analysis of the $\Delta L^{27}$; the values showed that the Premisa resin was more stable and that Beautifil II had also suffered major changes in both drinks. The different results observed among the composites is due to its composition, which differ from resin matrix composition, particle size and conversion after polymerization ${ }^{3}$. The resin matrix, which is responsible for the stability of color, can influence a higher staining ${ }^{25,28}$. Depending on the composition, it can absorb more or less water (and other substances), which leads to discoloration ${ }^{19}$. Another possibility is the presence of triethylene glycol dimethacrylate (TEGDMA), which can increase the hydrophilicity compared to urethane dimethacrylate (UDMA), and is more resistant to staining than bisphenol A glycidyl ether dimethacrylate (Bis-GMA) ${ }^{29}$. Thus, the color stability of Premisa resin can be justified by its complex composition of the resin matrix: Bis-GMA, BisEMA, and TEGDMA. Therefore, the unstable behavior of the Beautifil II can also be justified by its simplicity because of the presence of two resin monomers (Bis-GMA and TEGDMA).

Our results corroborates with Taşkinsel et al. ${ }^{19}$ (2014), which demonstrated a considerable color alteration of nano and micro hybrids composites resins when frequently immersed in sports drinks. In agreement with Mara da Silva et al. ${ }^{3}$ (2019), in this present study Beautifil II had the highest value of $\Delta E$ as well as the highest variation compared to Filtek Z350 XT. It was demonstrated that the consumption of beverages able to stain associated with brushing challenges leads to a decrease in microhardness, which was severer in Beautifil II; therefore, the surface treatments reduced the properties of Filtek Z350 XT and Beautifil II. In another study ${ }^{30}$ was observed that Beautifil II had the highest values for surface roughness after some superficial treatments. This fact can explain the considerable color alteration in the present study, since the surface roughness can influence the esthetic and biological outcomes of the composites ${ }^{30}$.

This study demonstrated the color alteration of composite resins induced by different solutions. This data complements the existing studies in the literature $\mathrm{s}^{3,8,10,11,30}$. Providing subsidies to conclude the different compositions of the resinous matrix can promote different results with natural or synthetic pigmentation drinks. Additionally, the drink's acidity can significantly alter the stability of the color of the composites, generating aesthetic disadvantages and disturbing clinical practice ${ }^{25}$. Thus, professionals 
should focus more attention to the different characteristics of each patient, such as their habits and customs, and then select the best restorative material.

More in vitro, ex vivo, and in vivo studies are needed for a greater understanding of the behavior of nanohybrid composite resins in the oral environment when in contact with solutions and commercially consumed drinks.

Considering the limitations of this study, it can be concluded that Premisa resin showed less change, while Beautifil II was more susceptible to staining. Among the beverages, the lemon flavor isotonic drink promoted major alterations.

\section{Acknowledgment}

This study was supported by CNPq through grant \#306516/2006-3 and PIBIC/USP.

\section{References}

1. Peutzfeldt A, Muhlebach S, Lussi A, Flury S. Marginal gap formation in approximal "Bulk Fill" resin composite restorations after artificial ageing. Oper Dent. 2018 Mar/Apr;43(2):180-9. doi: 10.2341/17-068-L.

2. Tsiagali V, Kirmanidou Y, Pissiotis A, Michalakis K. In vitro assessment of retention and resistance failure loads of teeth restored with a complete coverage restoration and different core materials. J Prosthodont. 2019 Jan;28(1):e229-6. doi: 10.1111/jopr.12668.

3. Mara da Silva T, Barbosa Dantas DC, Franco TT, Franco LT, Rocha Lima Huhtala MF. Surface degradation of composite resins under staining and brushing challenges. J Dent Sci. 2019 Mar;14(1):87-92. doi: 10.1016/j.jds.2018.11.005

4. Alharbi A, Ardu S, Bortolotto T, Krejci I. In-office bleaching efficacy on stain removal from CAD/CAM and direct resin composite materials. J Esthet Restor Dent. 2018 Jan;30(1):51-8. doi: 10.1111/jerd.12344.

5. Ceci M, Viola M, Rattalino D, Beltrami R, Colombo M, Poggio C. Discoloration of different esthetic restorative materials: A spectrophotometric evaluation. Eur J Dent. 2017 Apr-Jun;11(2):149-156. doi: 10.4103/ejd.ejd_313_16.

6. Oliveira DC, Souza-Júnior EJ, Prieto LT, Coppini EK, Maia RR, Paulillo LA. Color Stability and Polymerization Behavior of Direct Esthetic Restorations. J Esthet Restor Dent. 2014 JulAug;26(4):288-95. doi: 10.1111/jerd.12113

7. Lepri CP, Dibb RG. Surface roughness and color change of a composite: influence of beverages and brushing. Dent Mater J. 2012;31(4):689-96.

8. Lepri CP, Ribeiro MV, Dibb A, Palma-Dibb RG. Influence of mouthrinse solutions on the color stability and microhardness of a composite resin. Int J Esthet Dent. 2014 Summer;9(2):238-46.

9. Choi JW, Lee MJ, Oh SH, Kim KM. Changes in the physical properties and color stability of aesthetic restorative materials caused by various beverages. Dent Mater J. 2019 Feb 8;38(1):33-40. doi: 10.4012/dmj.2017-247.

10. Barbosa GF, Cardoso MB. Effects of carbonated beverages on resins composite stability. Am J Dent. 2018;31(6):313-6.

11. Rutkunas V, Sabaliauskas V, Mizutani H. Effects of different food colorants and polishing techniques on color stability of provisional prosthetic materials. Dent Mater J. 2010 Mar;29(2):167-76. 
12. Fujita M, Kawakami S, Noda M, Sano H. Color change of newly developed esthetic restorative material immersed in food simulating solutions. Dent Mater J. 2006 Jun;25(2):352-9.

13. Erdemir U, Yildiz E, Eren MM. Effects of sports drinks on color stability of nanofilled and microhybrid composites after long-term immersion. J Dent. 2012 Dec;40 Suppl 2:e55-63. doi: 10.1016/j.jdent.2012.06.002.

14. Antunes LS, Veiga L, Nery VS, Nery CC, Antunes LA. Sports drink consumption and dental erosion among amateur runners. J Oral Sci. 2017;59(4):639-43. doi: 10.2334/josnusd.16-0611.

15. Kumari RV, Nagaraj H, Siddaraju K, Poluri RK. Evaluation of the effect of surface polishing, oral beverages and food colorants on color stability and surface roughness of nanocomposite resins. J Int Oral Health. 2015 Jul;7(7):63-70.

16. Panahandeh $\mathrm{N}$, Torabzadeh $\mathrm{H}$, Naderi $\mathrm{H}$, Sheikh-Al-Eslamian SM. Effect of water storage on flexural strength of silorane and methacrylate-based composite resins. Restor Dent Endod. 2017 Nov;42(4):309-15. doi: 10.5395/rde.2017.42.4.309.

17. Tanthanuch S, Kukiattrakoon B, Siriporananon C, Ornprasert N, Mettasitthikorn W, Likhitpreeda S, et al. The effect of different beverages on surface hardness of nanohybrid resin composite and giomer. J Conserv Dent. 2014 May;17(3):261-5. doi: 10.4103/0972-0707.131791.

18. Miotti LL, Nicoloso GF, Durand LB, Susin AH, Rocha RO. Color stability of a resin composite: Effect of the immersion method and surface treatments. Indian J Dent Res. 2016 Mar-Apr;27(2):195-9. doi: 10.4103/0970-9290.183137.

19. Taşkinsel E, Ozel E, Ozturk E. Effects of sports beverages and polishing systems on color stability of different resin composites. J Conserv Dent. 2014 Jul;17(4):325-9. doi: 10.4103/0972-0707.136439.

20. Silva TMD, Sales ALLS, Pucci CR, Borges AB, Torres CRG. The combined effect of food-simulating solutions, brushing and staining on color stability of composite resins. Acta Biomater Odontol Scand. 2017 Jan 16;3(1):1-7. doi: 10.1080/23337931.2016.1276838.

21. Sedrez-Porto JA, Munchow EA, Cenci MS, Pereira-Cenci T. Translucency and color stability of resin composite and dental adhesives as modeling liquids - A one-year evaluation. Braz Oral Res. 2017 Jul 3;31:e54. doi: 10.1590/1807-3107BOR-2017.vol31.0054.

22. Gupta R, Parkash H, Shah N, Jain V. A spectrophotometric evaluation of color changes of various tooth colored veneering materials after exposure to commonly consumed beverages. J Indian Prosthodont Soc. 2005;5(2):72-8.

23. Ruyter IE, Nilner K, Moller B. Color stability of dental composite resin materials for crown and bridge veneers. Dent Mater. 1987 Oct;3(5):246-51.

24. Ferracane JL, Moser JB, Greener EH. Ultraviolet light-induced yellowing of dental restorative resins. J Prosthet Dent. 1985 Oct;54(4):483-7.

25. Batra R, Katarina P, Kapoor S. Effect of salivary ph on color stability of different flowable composites - a prospective in-vitro study. J Clin Diagn Res. 2016 Oct;10(10):ZC43-6.

26. Abed Kahnamouei M, Gholizadeh S, Rikhtegaran S, Daneshpooy M, Kimyai S, Alizadeh Oskoee P, et al. Effect of preheat repetition on color stability of methacrylate- and silorane-based composite resins. J Dent Res Dent Clin Dent Prospects. 2017 Fall;11(4):222-228. doi: 10.15171/joddd.2017.039.

27. Kotanidis A, Kontonasaki E, Koidis P. Color alterations of a PMMA resin for fixed interim prostheses reinforced with silica nanoparticles. J Adv Prosthodont. 2019 Aug;11(4):193-201. doi: 10.4047/jap.2019.11.4.193.

28. Dietschi D, Campanile G, Holz J, Meyer JM. Comparison of the color stability of ten new-generation composites: an in vitro study. Dent Mater. 1994 Nov;10(6):353-62. 
29. Bagheri R, Burrow MF, Tyas M. Influence of food-simulating solutions and surface finish on susceptibility to staining of aesthetic restorative materials. J Dent. 2005 May;33(5):389-98.

30. Ruivo MA, Pacheco RR, Sebold M, Giannini M. Surface roughness and filler particles characterization of resin-based composites. Microsc Res Tech. 2019 Oct;82(10):1756-1767. doi: 10.1002/jemt.23342. 